\title{
3D - food printing using extrusion: an insight
}

\begin{abstract}
Extrusion-based food printing unlike from food extrusion cooking, is a digitally-controlled extrusion process. The aim is to build up complex 3D food products layer by layer. Currently, it is one of the innovative method in the area of Food printing. The food printing can provide key solutions for digitalized food design as well as targeted nutrition control as per different needs and application. This mini review articles provides the glimpses of 3D Food printing specifically using Extrusion and also provides key information about the factors and the parameters associated with the extrusion and printing processes.
\end{abstract}

\author{
Volume 8 Issue $6-2018$
}

\begin{abstract}
Rakesh Mohan Shukla,' Madhuresh Dwivedi, ${ }^{2}$ Navneet Singh Deora, ${ }^{3}$ HN Mishra ${ }^{4}$, PS Rao ${ }^{4}$ 'Agricultural \& Food Engineering Department, IIT Kharagpur (WB), India

${ }^{2}$ Department of Food Process Engineering, National Institute of Technology, Rourkela, India

${ }^{3}$ Ingredients Innovation and Research, Jubilant Foodworks, India ${ }^{4}$ Agricultural \& Food Engineering Department, IIT Kharagpur (WB), India
\end{abstract}

Correspondence: Navneet Singh Deora, Innovation Scientist, Ingredients Innovation and Research, Jubilant Foodworks, India, Email navnetsinghdeora@gmail.com

Received: September 29, 2018 | Published: November 23 2018

\section{Introduction}

3D printing which is also known as additive manufacturing (AM) and rapid prototyping (RP), is an emerging digitalized technology. ${ }^{1-5}$ It is gaining extensive interest from researchers, industry and public with its diverse fields of applications in the areas such as medicine, gastronomy, engineering, manufacturing, art and education. With reference to the food sector, it will define innovative borders for food processing by being able to deliver a product that suits special consumer's criteria of taste, cost, convenience and nutrition. Therefore, although foods are complex systems with wide variations in physio-chemical properties, researchers have worked on widening the application of 3D printing to various types of food products.

Currently, consumers' attitudes for food decisions are decided by four criteria: taste, cost, experience, convenience and nutrition. However, in the recent time food market is growing for personalized healthy food. Another market which is coming up is personal gift market such as frosted patterns on biscuits and chocolates, letters carved into cookies, and logos painted onto food. Compared with foods manufactured in mass production, they are more nutrition controllable, but significantly more expensive and only available from very limited suppliers. ${ }^{6}$ Traditional mass food preparation processes, even those with advanced processing technologies, cannot meet such personalized demands Some food companies are exploring alternative food preparation methods to capture and maintain market share, in turn providing opportunities for food preparation methods such as three-dimensional (3D) printing. The next sections explains the application and benefits of the 3D Food Printing.

\section{Why print food, uses and benefits of 3D food printing}

One of the uses of 3D - Printing can be creating personalized food for a targeted consumer with specific medical conditions. For Example, 3D - printing can be used to develop soft, nutritious and innovative textured food for the elderly people with mastication and swallowing problems. Currently such elders address their nutrition needs from pureed food, most of which is unappealing and unappetizing. Thus, it can support to elderly group to cope up with their chewing and swallowing problems and provide better options. Another susceptible group that could benefit from personalizing food are children since they would be more willing to consume healthy nutritious snacks with innovative shapes, that could be used as educational tools as well. For examples, recent work published talks about the customized snack product using $3 \mathrm{D}$ printing technology to develop personalized food formula, a fruit based snack for children. Lille et al. ${ }^{4}$ have designed a 3D printed customized healthy snack made of protein, starch and fiber rich materials that are considered nutritive functional ingredients. These personalized foods for health form the next logical step in personalizing the quality of life.

3D printing can bridge the gap between culinary arts and nonprofessional food artisans by decreasing the cost of production. Another example could be in the area of Novel Food Structuring Using a Broad Range of Alternative Food Ingredients. Finally, 3D printing can provide Environmentally Friendly and Sustainable Technology and promoting Higher Social Bonding through Food Messaging.

\section{Food 3D printing}

$3 \mathrm{D}$ printing in the area of food has seen recent upsurge. In one of the patented work, 3D printing was used in development of cake Mix by application of paste extrusion technique by the use of mixture of starch, sugar, corn syrup, yeast and a cake frosting. ${ }^{1}$ Foods created by $3 \mathrm{D}$ printing have already entered the market, although most consumers may still be unaware. For examples, in Netherlands, 3D printing is being used for the application of microwave pancake fabrication. Different methods are employed for 3D Food printing such as selective laser sintering/hot air sintering, hot-melt extrusion/ room temperature extrusion, binder jetting, and ink jet printing. Among the available methods, extrusion-based 3Dfood printing is the one of the most widely adopted and applied method. As shown in Figure 1 below, the current extrusion-based food printing starts with designing a virtual 3D model. Slicing software than translates this model into individual layer pattern. This than generates machine codes for printing. Once the codes are uploaded into a printer, preferred food recipe is introduced and after this food printing process 
in initiated. As per the layer patterns generated from the 3D model, the extruded material is dispensed either by moving the nozzle above a motorized stage or by moving the stage underneath the nozzle to form a layer. Each layer welds to the previous layer on the stage, and forms a layer based 3D structure. The printed foods may go through a post-deposition cooking process such as baking as per requirement and target application.

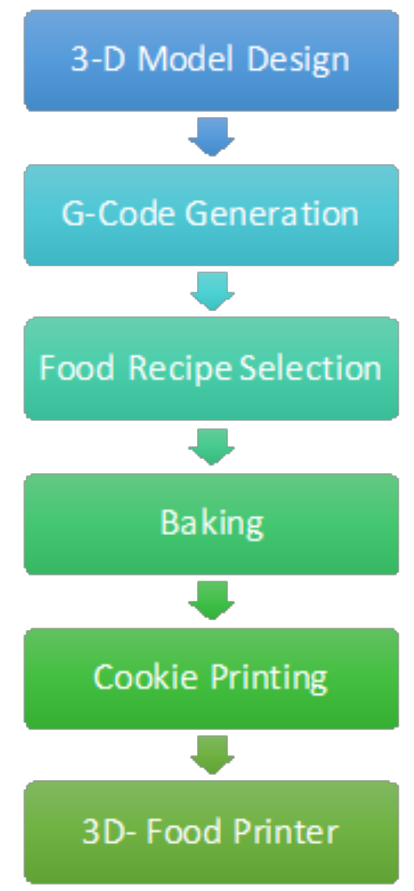

Figure I Process flow sheet for 3 D Food Printing.
A number of articles in the area of 3D food Extrusion have been published. ${ }^{1-6}$ Most of this work focuses on the fabrication of customized food items, recipe modification, food printing process control and equipment modification. Researchers from Netherlands Organization for Applied Scientific Research (TNO) have started to explore more fundamental topics such as converting ingredients into tasty products for health and economic concerns by the use of $3 \mathrm{D}$ Printing application.

\section{Extrusion in 3D food printing}

Extrusion process normally uses force to pass materials through a die opening thereby creating different products/objects as per intended requirement and application. In terms of similarity, Food extrusion cooking and 3D food printing using extrusion can automate a Food preparation with improved efficiency in the process resulting in the enhancement of the food quality. Extrusion-based 3D food printing aims to achieve the output of the conventional food extrusion processing physically with a digitalized design and a personalized nutrition control. In terms of the user experience, both the methods could have different outlook. 3D Printing using extrusion is a digitally-controlled, robotic construction process which aims to build up complex 3D food products layer by layer. ${ }^{7,8}$ It initiates with material loading and thereafter pushing the material out of the nozzle in a controlled manner, moving the material stream according to predefined path, and eventually bonding the deposited layer to form a coherent solid structure. The extrusion-based food printer consists of a multi-axis stage and one or more extrusion units. With the assistance of computer and algorithms, such advanced printers can manipulate food fabrication in real. Figure 2 below provides overview of the mechanism, Process parameters and design used in the Extrusion 3D Printing. ${ }^{9-12}$

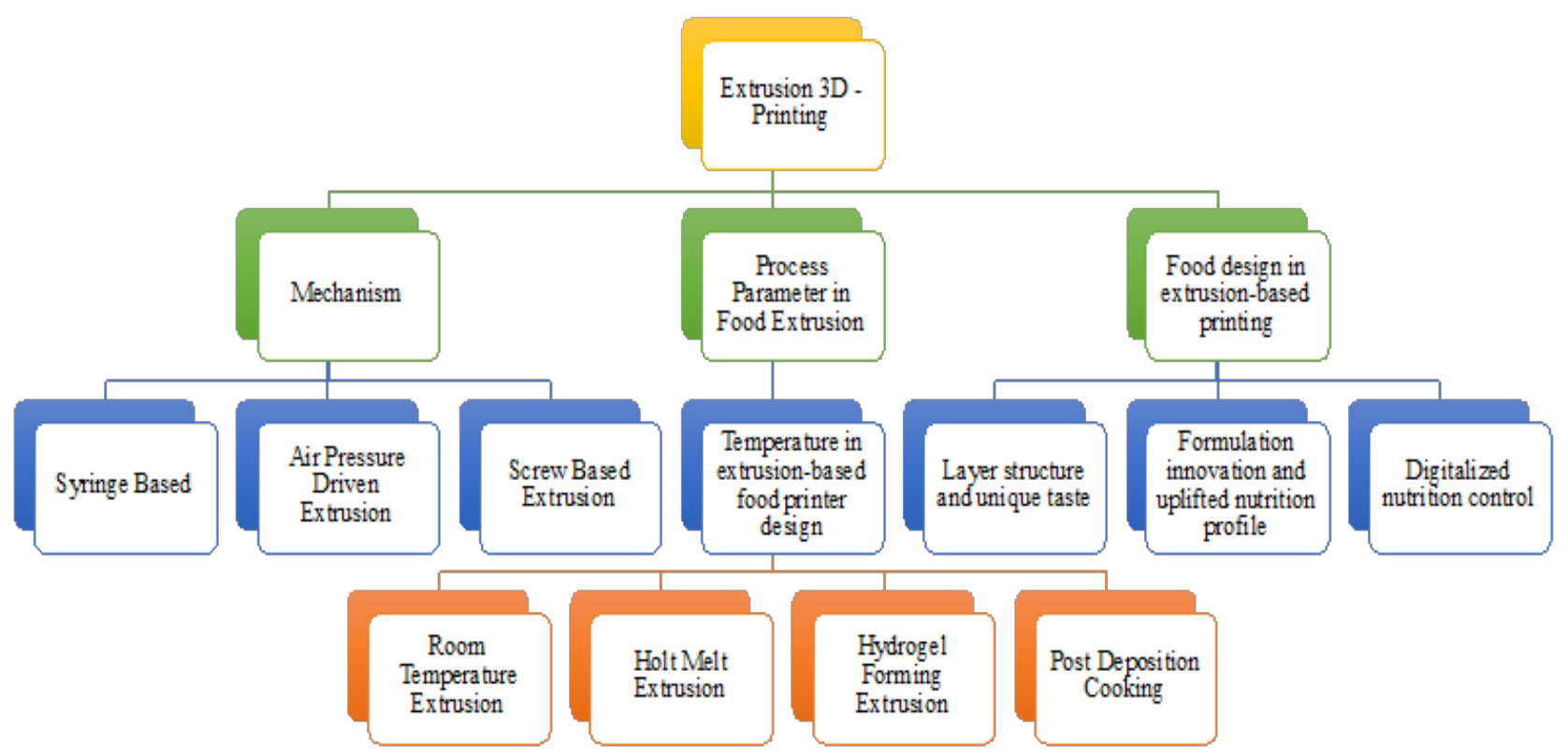

Figure 2 Extrusion 3D Printing - mechanism, process and food design. 


\section{Future update}

In the recent time, many companies and research institutes are working on improving the extrusion-based food printing technology with the intend to commercialize it and thereby promoting innovative design and healthy life style. However, the current available applications are still in the primitive stage and would need and investigation to reach at the commercial level. The application of $3 \mathrm{D}$ printing technology should enable in future to use alternative ingredients with enhanced product with respect to the nutritional content.

\section{Acknowledgments}

None.

\section{Conflicts of interest}

The authors declare that there is no conflicts of interest.

\section{References}

1. Yang J, Wu L, Liu J. Rapid prototyping and fabrication method for 3-Dfood objects, 2001; US Patent No. 6280785.

2. Jie Sun, Weibiao Zhou, Liangkun Yan, et al. Extrusion-based food printing for digitalized food design and nutrition control. Journal of Food Engineering. 2018;220:1-11.

3. Tan Cavin. Extrusion-based 3D food printing-Materials and machines. International Journal of Bioprinting. 2018;4:2.
4. Lille M, Nurmela A, Nordlund E, et al. Applicability of protein and fiber-rich food materials in extrusion-based 3D printing. Journal of Food Engineering. 2018;220:20-27.

5. Zhenbin L, Zhang M, Bhandari B, et al. Impact of rheological properties of mashed potatoes on 3D printing. Journal of Food Engineering. 2018;220:76-82.

6. Zhao Haiming, Jufeng Wang, Xiaoyu Ren, et al. Personalized food printing for portrait images. Computers \& Graphics. 70 (2018):88-197.

7. Vancauwenberghea V, Mfortaw Mbonga VB, Vanstreels E, et al. 3D printing of plant tissue for innovative food manufacturing: Encapsulation of alive plant cells into pectin based bio-ink. Journal of Food Engineering. 2017.

8. Yang Fan, Min Zhang, Bhesh Bhandari. Recent development in 3D food printing. Critical reviews in food science and nutrition. 2017;57(14):31453153.

9. Wang L, Zhang M, Bhandari B, et al. Investigation on fish surimi gel as promising food material for 3D printing. Journal of Food Engineering. 2018;220:101-108.

10. Sun Jie, Weibiao Zhou, Dejian Huang, et al. 3D Food Printing: Perspectives. Polymers for Food Applications. 2018;725-755.

11. Makino Masato, Daisuke Fukuzawa, Takahiro Murashima, et al. Simulation of 3D food printing extrusion and deposition. Nanosensors, Biosensors, Info-Tech Sensors and 3D Systems. 2017;10167.

12. Dunham Simon. Applications of 3D Printing. 3D Printing Applications in Cardiovascular Medicine. Academic Press, 2018;61-78. 